\title{
A prospective Study on Wrist Tuberculosis: Challenges in Diagnosis and Management
}

\author{
Ram K Shah ${ }^{1 *}$, Ankit Shrivastava ${ }^{2}$, Sujan R Paudel ${ }^{3}$ and Rajesh C Das ${ }^{4}$ \\ ${ }^{1}$ Department of Orthopaedics, Janaki Medical College, Nepal \\ ${ }^{2}$ Department of Orthopaedic Surgeon, Janakpur Trauma Hospital, Nepal \\ ${ }^{3}$ Consultant Orthopaedic Surgeon, Scheer Memorial Adventist Hospital, Nepal \\ ${ }^{4}$ Department of Medicine, Janaki Medical College, Nepal
}

*Corresponding author : Ram K Shah, Department of Orthopaedics, Janaki Medical College, Janakpurdham, Nepal.

Received Date: August 28, 2019

Published Date: September 13, 2019

\section{Abstract}

Introduction: Wrist Joint is an uncommon site for tubercular infection. However, it is more commonly seen in south-east Asia than in developed parts of the world. The diagnosis is often delayed, because of its rarity and hence many patients have to live with long term sequale of stiffness and pain.

Materials and Methods: Twenty-two (22) patients presenting with wrist joint tuberculosis between January 2013 and January 2018 were treated with antitubercular drugs, splinting and were prospectively followed up for a minimum of 18 months duration. The evaluation of outcome was based on Patient Rated Wrist Evaluation.

Result: Seventeen (17) patients had microbiological or histopathological confirmation of diagnosis. Five (5) patients with high clinical suspicion and negative diagnostic workup were also treated for tubercular arthritis. Mean Patient Rated Wrist Evaluation improved from 115 at the beginning to 31 at 18 months.

Conclusion: It is important to avoid delay in the diagnosis of wrist tuberculosis. Whenever the clinical suspicion is high and diagnostic workup is negative, a therapeutic trial of anti-tubercular drugs could be option to escape and manage this havoc early. Chemotherapy with combination of anti-tubercular drugs is safe and effective in treatment of wrist tuberculosis.

\section{Introduction}

Musculoskeletal Tuberculosis (TB) is the third most frequent site of extrapulmonary tuberculosis after the pleura and lymph nodes [1]. Although musculoskeletal TB has become uncommon in the Western world, it remains a huge problem in Asia, Africa, and many developing countries [2]. One third of TB cases worldwide are reported from south-east Asia [3].

Mycobacterium tuberculosis can infect any bone, joint, tendon, or bursa. TB of peripheral joints occur infrequently, but if untreated, it can cause serious joint destruction as well as spread of the infection to the surrounding bursa, muscles, tendons and other soft tissues [4]. The reported incidence of wrist TB is less than $1 \%$ of all osteoarticular TB [5]. Although rare, TB of the wrist is a cause of major morbidity [5,6]. The diagnosis of wrist TB is mostly missed. The delay in diagnosis is because of minimal initial symptoms, rarity of the lesion and ability of wrist TB to mimic more common pathologies [5]. Late diagnosis may result in bone damage and major morbidity [7]. Even after treatment, many patients have to continue with stiffness and pain [8]. Early diagnosis of bone and joint disease is important to minimize the risk of deformity and enhance outcome [1].

\section{Materials and Methods}

A total of 28 patients with tubercular arthritis of wrist, presented to Janakpur Trauma Hospital between January 2013 and January 2018. Of the 28 patients, six were with follow up less than 18 months and, were excluded from our study. In total, 22 patients were enrolled in this study. Patients presenting with problems of swelling and monoarticular pain of the wrist were enrolled in this study. Appropriate history was taken, and examination was done. 
Swelling, tenderness and range of motion were noted. Regional lymph nodes including axillary, supratrochlear and epitrochlear lymph nodes were examined. Skin lesions and ocular signs were examined to rule out other rheumatological conditions.

Laboratory studies including complete blood counts (CBC), erythrocyte sedimentation rate (ESR), C-reactive protein (CRP) and Mantoux test were done. Sputum for acid fast bacilli and chest $\mathrm{X}$-ray was done in all patients to look for coexisting pulmonary TB. Screening for HIV was done to diagnose TB-HIV coinfection. Synovial fluid was aspirated in 8 patients and sent for Gram stain,
Zeehl Nelson stain, aerobic culture, PCR and Culture in Lowenstein Johnson media. Patients with suspicious X-ray and negative microbiological analysis were sent for magnetic resonance imaging (MRI). Synovial biopsy of the wrist was done under regional nerve block in 5 patients and sent for histopathology study. nti-tubercular Therapy (ATT) including Isoniazid, Rifampicin, Ethambutol and Pyrazinamide was started after appropriate investigations were available. 5 patients with clinical suspicion of wrist TB and negative diagnostic workup were subjected to a therapeutic trial of ATT (Figure 1).

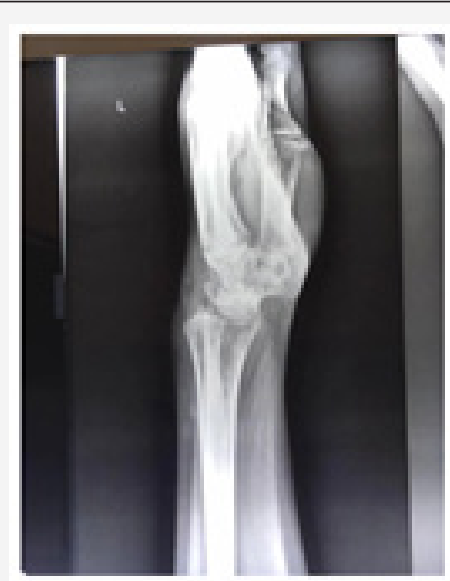

Figure 1: Radiological picture before treatment.

All patients were enrolled in National Tuberculosis Programme of Nepal and received free ATT as per National Tuberculosis Center's Community Based Directly Observed Treatment Shortcourse (DOTS) strategy [9]. CBC, ESR and CRP levels were monitored at 2 months and every 3 months afterwards until ESR and CRP were normal. After 2 months of 4 drug therapy, patients were started on 2 drug therapy including isoniazid and rifampicin for another 6-10 months. Once pain was relieved and ESR was almost normal, the entire drug was also stopped (around 8-12 months). Splints or plaster slabs were used for initial periods to alleviate pain, usually for a period of 4 weeks. Follow-up radiographies were performed at $3,6,12$ and 18 months and then every year thereafter.

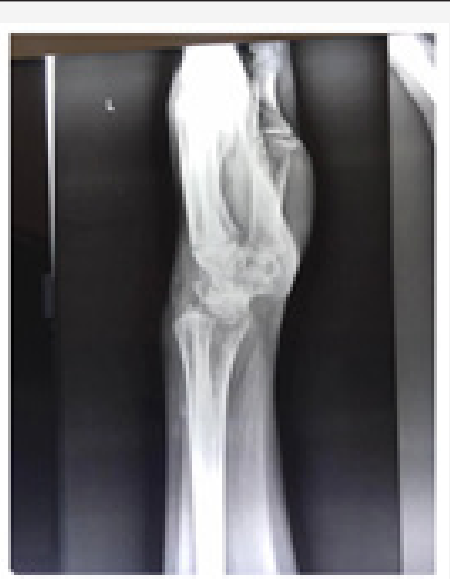

The Patient Rated Wrist Examination (PRWE) [10] questionnaire is a subjective outcome measure consisting of 15 questions answered on a scale of 1 to 10 . Five questions focus on wrist pain, and ten questions focus on function (Figure 4). A score out of 150 is obtained with zero being asymptomatic and 150 implying complete functional loss with severe pain. The score achieved on each patient's questionnaire was recorded at 3, 6, 12 and 18 months. All patient's data was enterd in the SPSS software and analysed using paired sample t-test for patient rated wrist evaluation and ESR values before treatment and after treatment. $P$ value less than 0.05 was considered significant statistically (Figure 2-4).
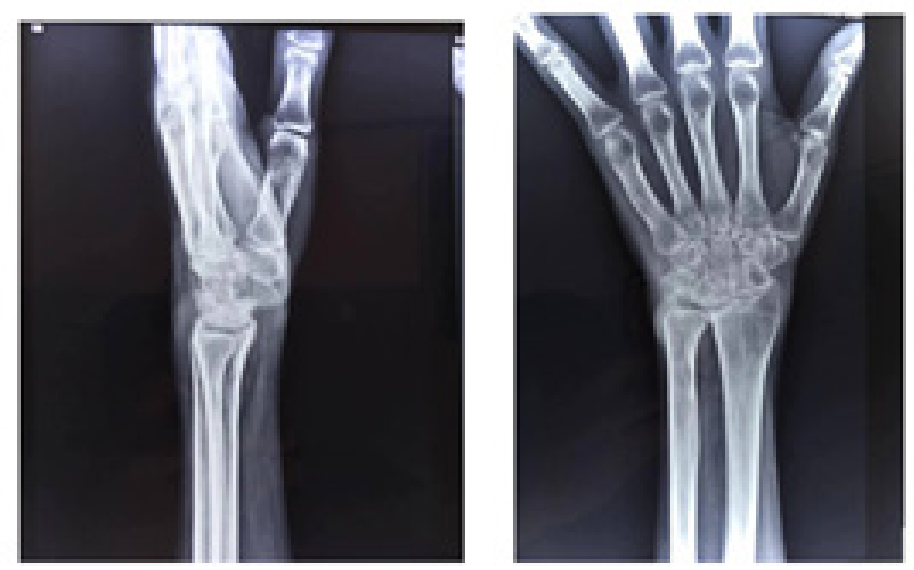

Figure 2: Radiological pictures after 2 months during treatment. 


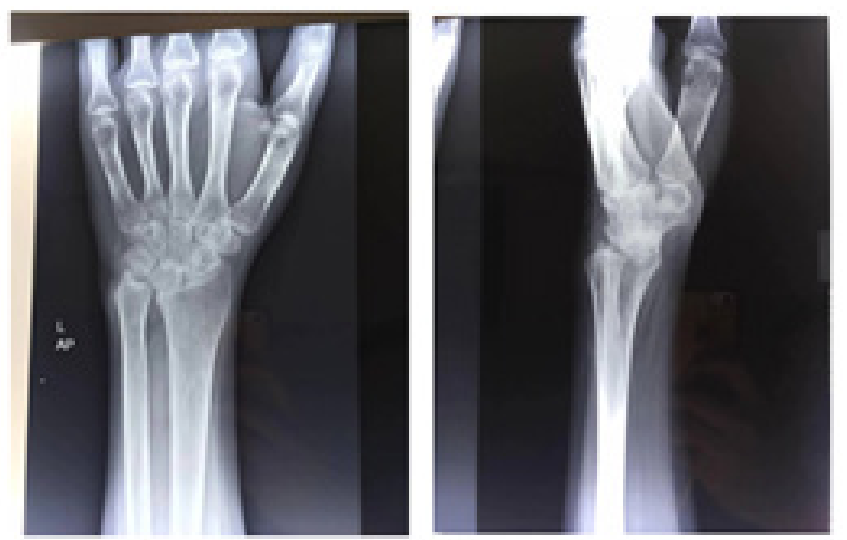

Figure 3: Radiological pictures after 8 months of therapy.

\section{PATIENT-RATED WRIST EVALUATION}

Name

Date

The questions below will help us understand how much difficulty you have had with your wrist in the past week. You will be describ. ing your average wrist symptoms over the past week on a scale of 0 to 10 . Please provide an answer for ALL questions. If you did not perform an activity, please ESTIMATE the pain or difficulty you would expect. If you have never performed an activity, you may leave it blank.

\section{PAIN}

Rate the average amount of pain in you wrist over the past week by circling the number that best describes your pain on a scale from 0 to 10. A zero (0) means that you did not have any pain and a ten (I0) means that you had the worst pain you have ever experienced or that you could not do the activity because of pain.

$$
\begin{array}{lccccccccccc}
\text { Sample scale: } & \begin{array}{cccccccc}
0 \\
\text { No Pain }
\end{array} & 1 & 2 & 3 & 4 & 5 & 6 & 7 & 8 & 9 & 10 \\
\text { Worst Ever }
\end{array}
$$

RATE YOUR PAIN:

At rest

When doing a task with a icpeated wrist movement

When lifting a heavy object

When it is at its worst

How often do you have pain?

$\begin{array}{ccccccccccc}0 & 1 & 2 & 3 & 4 & 5 & 6 & 7 & 8 & 9 & 10 \\ 0 & 1 & 2 & 3 & 4 & 5 & 6 & 7 & 8 & 9 & 10 \\ 0 & 1 & 2 & 3 & 4 & 5 & 6 & 7 & 8 & 9 & 10 \\ 0 & 1 & 2 & 3 & 4 & 5 & 6 & 7 & 8 & 9 & 10 \\ 0 & 1 & 2 & 3 & 4 & 5 & 6 & 7 & 8 & 9 & 10 \\ \text { Never } & & & & & & & & & & \text { Alwayz }\end{array}$

\section{FUNCTION}

\section{A. SPECIFIC ACTIVITIES}

Rate the amount of difficulty yu experienced performing each of the tiems lasied beiow, uver the pusi week, by curcling the: number that describes your difficulty on a scale of 0 to 10 . A zero (0) means you did not experience any difficulty and a ten. (10) means it was so difficult you were unable to do it at all,

Sample scale:

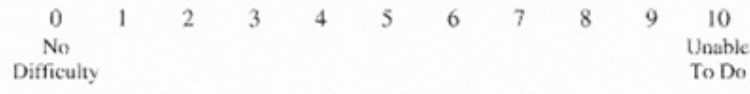

I urn a door knob using my affected hand

Cut meat using a knife with my affected hand

Fasten buttons on my shirt

Use my affected hand to push up from a chair

Carry a 10-pound object in my affected hand

Use bathroom tissue with my affected hand

$\begin{array}{lllllllllll}0 & 1 & 2 & 3 & 4 & 5 & 6 & 7 & 8 & 9 & 10 \\ 0 & 1 & 2 & 3 & 4 & 5 & 6 & 7 & 8 & 9 & 10 \\ 0 & 1 & 2 & 3 & 4 & 5 & 6 & 7 & 8 & 9 & 10 \\ 0 & 1 & 2 & 3 & 4 & 5 & 6 & 7 & 8 & 9 & 10 \\ 0 & 1 & 2 & 3 & 4 & 5 & 6 & 7 & 8 & 9 & 10 \\ 0 & 1 & 2 & 3 & 4 & 5 & 6 & 7 & 8 & 9 & 10\end{array}$

\section{B. USUAL ACTIVITIES}

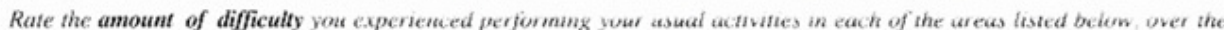
past week, by circling the number that best describes your difficulty on a scale of o to 10. By "usual activities," we mean the activities you performed before you started having a problem with your wrist. A zero $(0)$ means that you did not experience any difficulty and a ten (10) means it was so difficult you were unable to do any of your usual activities.

$\begin{array}{lllllllllllll}\text { Personal care activities (dressing, washing) } & 0 & 1 & 2 & 3 & 4 & 5 & 6 & 7 & 8 & 9 & 10\end{array}$

$\begin{array}{llllllllllll}\text { Household work (cleaning, maintenance) } & 0 & 1 & 2 & 3 & 4 & 5 & 6 & 7 & 8 & 9 & 10\end{array}$

$\begin{array}{llllllllllllll}\text { Work (your job or usual everyday work) } & 0 & 1 & 2 & 3 & 4 & 5 & 6 & 7 & 8 & 9 & 10\end{array}$

$\begin{array}{lllllllllllll}\text { Recreational activities } & 0 & 1 & 2 & 3 & 4 & 5 & 6 & 7 & 8 & 9 & 10\end{array}$

Figure 4: Patient rated wrist evaluation (PRWE) chart. 


\section{Result}

28 patients with tubercular arthritis of wrist were seen during the study period of five years. 22 completed follow up upto 18 months and were included in this study. 14 of them were males (64\%) and 8 were females (36\%). 13 were in 20-30 years age group (59\%), 6 in 30-40 years age group (27\%) and 3 were more than 60 years age (14\%). 19 patients were right dominant (86\%) and 3 were left dominant (14\%). Dominant hand was affected in 14 patients (63\%). The most common presenting complaint was pain, followed by stiffness of the wrist. Discharging sinus was not seen in any cases. Five of them had positive contact history with TB patient. Elevation of ESR more than $50 \mathrm{~mm}$ in first hour was the most sensitive test (seen in $86 \%$ ). Total leucocyte count was normal in 14 patients (63\%). Leukocytosis (white blood cells more than 11000 cells $/ \mathrm{ml}$ ) was seen in 7 patients (32\%). Aerobic culture showed growth of staphylococcus in 6 patients (27\%).

Juxtaarticular osteoporosis was the most common x-ray finding, seen in 20 patients (91\%) followed by soft tissue swelling in 10 (45\%), cystic changes in 9 and gross destruction of articular surface in 3 cases. MRI was done in 12 patients and most commonly showed synovial hypertrophy followed by cartilage erosion, tenosynovitis and exudates in the joint space. PCR of aspirated synovial fluid was done in 8 patients and found to be positive for mycobacterium tuberculosis in 5 patients.

Synovial biopsy was done in 5 patients (only when other investigations were inconclusive) and showed classical caseative necrosis with langerhan's giant cells and lymphocytic infiltration in all 5 patients. HIV coinfection was seen in 3 patients (13.6\%). These cases were managed conservatively with appropriate ATT, Antiretroviral therapy and splints. Concurrent pulmonary tuberculosis was seen in 7 patients, who were treated with ATT. The duration of symptoms ranged from 6 weeks to 28 weeks (mean duration was 16 weeks). The time from presentation to the initiation of ATT was 1-2 weeks. 2 patients discontinued ATT due to serious hepatotoxicity and restarted after 2 weeks at lower dose. They tolerated it and continued until clinical resolution. None were resistant to first line antitubercular therapy.

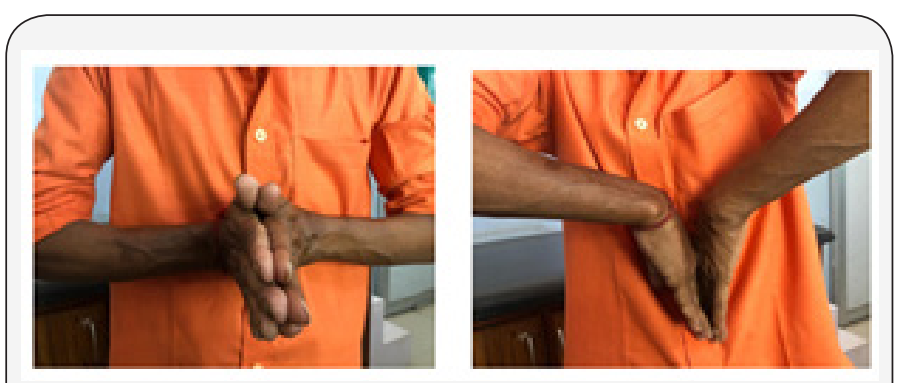

Figure 5: clinical pictures after 8 months of therapy.

The mean PRWE at presentation was 115 at presentation which decreased to 82 at 3 months, 67 at 6 months, 49 at 12 months and 31 at 18 months. The improvement in PRWE was accompanied by fall in CRP and ESR values during consecutive follow up visits. Paired sample t-test showed that the improvement in PRWE at presentation and final follow up at 18 months is statistically significant ( $p$ value $=0.02$ ). The subgroup of 5 patients where ATT was started on clinical basis alone also showed similar PRWE scores as other 17 patients. All patients were assessed for clinical improvements (Figure 5).

\section{Discussion}

TB is a major health issue in developing countries, with over 2 billion people being infected with M. tuberculosis worldwide.[3] As per the WHO bulletin 2010 most cases were reported in the middle age group. Men were 1.73 times more commonly affected than women [1]. In our study the male to female ratio was 1.77 and $86 \%$ were in the middle age group (20-39 years). As shown in existing literature, dominant side was more commonly affected than nondominant side [11]. Around 9.9\% of HIV is co-infected with TB and around $2.4 \%$ of TB with HIV in Nepal [12]. HIV coinfection in our study population was $14 \%$.

Tuberculous arthritis is known to occur commonly in the weight-bearing joints, particularly the vertebral joints, followed by the hip and knee joints [13]. It is not uncommon to see rare forms of tubercular disease in endemic zones. Lertsrisatit $\mathrm{P}$ et al in a study of 27 extraspinal TB found wrist TB to be the second most common next to knees [14]. Though monoarticular TB of wrist has been described as a rare entity by many European authors, most of our patients had monoarticular involvement $[6,15,16]$. Tuberculous infection of joints follows either direct invasion from an adjacent area of tuberculous osteomyelitis or from haematogenous spread. Quiescent tuberculosis can be reactivated after an episode of minor trauma [17].

Diagnosis of any extrapulmonary tuberculosis is usually confirmed by the pathological examination of biopsy material and positive culture. It is well known that the frequent absence of laboratory assistance complicates the diagnosis of the disease [18]. Recent history of contact with TB patients, and the presence of constitutional symptoms like weight loss, loss of apetite, night sweat, evening rise of temperature support the diagnosis of TB [19]. However, constitutional symptoms are seen in only one third of musculoskeletal tuberculosis patients [20].

A mean delay of 16 weeks until diagnosis was seen in our cases. It's because the clinical presentation mimics other conditions like chronic pyogenic osteomyelitis, broodie's abscess, or tumours $[21,22]$. Sbai et al, while doing excision of a ganglion found a pseudotumoral mass with rice bodies. The histopathological study revealed caseating granulomas. Cultures on LöwensteinJensen medium detected Mycobacterium tuberculosis [23]. Even a synovial fluid analysis suggestive of pseudogout cannot exclude the chance of articular tuberculosis, coexistence of these two chronic arthritic conditions in wrist has been reported by Watanabe et al. [24] Moreover, late diagnosis leads to poor functional outcome even in the presence of regular ATT [25].

Blood test markers like ESR and CRP are elevated in many pathological conditions like inflammation and infection. Radiological findings of periarticular osteoporosis, soft tissue 
swelling and destruction of joint architecture with loss of joint space are findings of any chronic arthritis and equally mimics TB findings [2]. Although imaging features of TB of joints and tendons are nonspecific, certain findings such as relatively preserved joint space, juxta-articular osteoporosis, cold abscesses, para-articular soft tissue calcification, and rice bodies are suggestive of TB infection [4]. Common X-ray findings for wrist TB include: (1) bone atrophy, (2) bone destruction by "honeycombing" or cystic lesions with or without sequestrate, (3) little or no periostitis [2].

Mycobacterium tuberculosis is not always detected in specimens. Direct smear examination of the pathological material might reveal acid-fast bacilli in the synovial fluid in $10 \%$, in the synovial tissue in $20 \%$, and in the regional lymph node in $30 \%$ of tuberculous arthritis [26]. Diagnosis is often established after direct microscopic examinations, a PCR assay for M. tuberculosis, and a Mycobacterium culture from cold abscess aspiration fluid. PCR is particularly useful as it uses nucleic acid amplification, is highly sensitive for detecting M. tuberculosis, and takes only few hours for confirmation. If these examinations are negative, a surgical biopsy is recommended [11].

In patients from $\mathrm{TB}$ endemic areas, where there is high index of suspicion and negative diagnostic workup, a therapeutic trial of ATT drugs can be given for 4 weeks. Improvement of symptoms after ATT is also suggestive for making a diagnosis of tuberculosis $[27,28]$. ATT was started in our 5 patients without evidence to confirm the diagnosis. This subgroup of patients also improved similar to other patients. As in the series by Kotwal et al, most of our patients responded favourably to conservative management [25]. Though some residual stiffness was present in few cases, there was no functional limitation.

Anti-tuberculosis treatment includes a cocktail of antibiotics, namely Isoniazid, Rifampicin, Ethambutol and Pyrazinamide which are bactericidal and bacteriostatic against M. tuberculosis [29]. A total of 6 to 9 months of a rifampicin-based regimen, like treatment of pulmonary TB, is recommended for the treatment of drug susceptible musculoskeletal disease [1]. Prognosis is good with chemotherapy and non-operative management [30]. Surgery should be reserved for specific indications, namely a biopsy, debridement, fusion of a disorganized joint or carpal tunnel decompression. Surgery may also be indicated in a patient who does not initially show a good response to ATT. The local blood flow is increased following surgery, resulting in early healing [31]. Because smear microscopy or culture is not available to monitor patients with extrapulmonary $\mathrm{TB}$, clinical monitoring is the usual way to assess the response to treatment [19]. Limitation of the study was failure to undertake all diagnostic procedures in all patients. This would have helped us to calculate the diagnostic accuracy of each procedure.

\section{Conclusion}

Tuberculosis of the wrist is one of the rare forms of Extrapulmonary TB. South-east-Asia being endemic region, many such cases are missed or misdiagnosed. It is important to avoid delay in diagnosis and decrease in the long-term morbidity. Whenever clinical suspicion is high and diagnostic workup is negative, a therapeutic trial of anti-tuberculosis drugs could be option to escape and manage this havoc early. Chemotherapy with combination of antitubercular drugs is safe and effective in treatment of wrist tuberculosis.

\section{Acknowledgement}

None.

\section{Conflict of Interest}

No conflict of interest.

\section{References}

1. Leonard MK, Blumberg HM (2017) Musculoskeletal Tuberculosis. Microbiol Spectr 5(2).

2. De Backer AI, Vanhoenacker FM, Sanghvi DA (2009) Imaging features of extraaxial musculoskeletal tuberculosis. Indian J Radiol Imaging 19(3): 176-86.

3. Nair NF, Wares, Sahu S (2010) Tuberculosis in the WHO South-East Asia Region. Bull World Health Organ 88(3): 164.

4. Pattamapaspong N, Muttarak M, Sivasomboon C (2011) Tuberculosis arthritis and tenosynovitis. Semin Musculoskelet Radiol 15(5): 459-469.

5. Prakash J, Mehtani A (2017) Hand and wrist tuberculosis in paediatric patients - our experience in 44 patients. J Pediatr Orthop B 26(3): 250260.

6. Palma J, Díaz E, Islas S, Silva J, Mella S (2017) Monoarticular tuberculosis of the wrist: a case report. Rev Chilena Infectol 34(5): 511-515.

7. Aghoutane EM, Salama T, El Fezazi R (2017) Tuberculosis of the wrist in children: A rare manifestation. Int J Mycobacteriol 6(1): 106-107.

8. Benchakroun M, El Bardouni A, Zaddoug O, Kharmaz M, El Yaacoubi M et al (2004) Tuberculosis of the wrist. Symptoms and outcome in eleven cases. Rev Chir Orthop Reparatrice Appar Mot 90(4): 337-345.

9. https://nepalntp.gov.np/wp-content/uploads/2019/01/CB-DOTSGuideline-2073.pdf

10. Mac Dermid JC, Turgeon T, Richards RS, Beadle M, Roth JH et al. (1998) Patient Rating of Wrist Pain and Disability: A Reliable and Valid Measurement Tool. Journal of Orthopaedic Trauma 12(8): 577-586.

11. Lee KE, (1985) Tuberculosis presenting as carpal tunnel syndrome. J Hand Surg Am 10(2): 242-245.

12. Suvedi B, (2003) HIV-TB co-infection in Nepal. J Inst Med 25: 19-21.

13. Karakaplan M, Köroğlu M, Ergen E, Aslantürk O, Özdemir ZM et al. (2017) Isolated Tuberculosis of Capitate and Triquetrum. J Wrist Surg 6(1): 70-73.

14. Lertsrisatit P, Nantiruj K, Totemchokchyakarn K, Janwityanujit S (2007) Extraspinal tuberculous arthritis in HIV era. Clin Rheumatol 26(3): 319321.

15. Sbai MA, Benzarti S, Bouzaidi K, Sbei F, Maalla R (2015) A rare localization of tuberculosis of the wrist: The scapholunate joint. Int J Mycobacteriol 4(2): 161-164.

16. Torres Lozano P, Gallach Sanchis D, Pardo Coello MM (2012) Osteoarticular tuberculosis with destructive wrist arthritis secondary to extrapulmonary tuberculosis. Rev Esp Cir Ortop Traumatol 56(5): 37880.

17. Jeong GK, Lester B (2001) Mycobacterium tuberculosis infection of the wrist. Am J Orthop (Belle Mead NJ) 30(5): 411-414.

18. Luk KD (1999) Tuberculosis of the spine in the new millennium. Eur Spine J 8(5): 338-345. 
19. Lee JY (2015) Diagnosis and treatment of extrapulmonary tuberculosis. Tuberc Respir Dis (Seoul) 78(2): 47-55.

20. Cormican L, Hammal R, Messenger J, Milburn HJ (2006) Current difficulties in the diagnosis and management of spinal tuberculosis. Postgrad Med J 82(963): 46-51.

21. Desikan P, Rahul Verma, Karuna Tiwari, Nikita Panwalkar (2016) Acute Monoarthritis of the Wrist Joint: Tuberculosis or Not? J Wrist Surg 5(1): 77-79.

22. Higuchi S, Ishihara S, Kobayashi H, Arai T (2008) A mass lesion of the wrist: a rare manifestation of tuberculosis. Intern Med 47(4): 313-316.

23. Sbai MA, Benzarti S, Msek H, Boussen M, Khorbi A (2016) Pseudotumoral form of soft-tissue tuberculosis of the wrist. Int J Mycobacteriol 5(1): 99101.

24. Watanabe KY, Yamazaki, Sugawara M (2017) Tuberculosis of the Wrist Accompanied with Calcium Pyrophosphate. Intern Med 56(24): 33893394.

25. Kotwal PP, Khan SA (2009) Tuberculosis of the hand: clinical presentation and functional outcome in 32 patients. J Bone Joint Surg Br 91(8): 10541057.
26. Tuli SM (1997) Tuberculosis of the skeletal system. Jaypee, Delhi.

27. Pauker SG, Kassirer JP (1980) The threshold approach to clinical decision making. N Engl J Med 302(20): 1109-1117.

28. Khan R, Abid S, Jafri W, Abbas Z, Hameed K, et al. (2006) Diagnostic dilemma of abdominal tuberculosis in non-HIV patients: an ongoing challenge for physicians. World J Gastroenterol 12(39): 6371-6375.

29. Sotgiu G, Rosella Centis, Lia Dambrosio, Giovanni Battista Migliori (2015) Tuberculosis treatment and drug regimens. Cold Spring Harb Perspect Med 5(5): a017822.

30. Harish S Hosalkar, Nina Agrawal, Swapna Reddy, Kriti Sehgal, Edward J Fox et al, (2009) Skeletal tuberculosis in children in the Western world: 18 new cases with a review of the literature. Journal of Children's Orthopaedics 3(4): 319-324.

31. Tuli SM (1993) Tuberculosis of the skeletal system: bone, joints, spine, and bursal sheaths. First ed. New Delhi: Jaypee Brothers Medical Publishers 116-17. 